\title{
TRIM39-RPP21 Variants ( $\triangle 19$ InsCCC) Are Not Associated with Juvenile Idiopathic Epilepsy in Egyptian Arabian Horses
}

\author{
Victor N. Rivas ${ }^{1}$, Monica Aleman ${ }^{2}{ }^{\circledR}$, Janel A. Peterson ${ }^{1}$, Anna R. Dahlgren ${ }^{1}$, Erin N. Hales ${ }^{1}$ \\ and Carrie J. Finno ${ }^{1, *(\mathbb{D})}$ \\ 1 Department of Population, Health and Reproduction, University of California, Davis, CA 95616, USA; \\ vnrivasgutierrez@ucdavis.edu (V.N.R.); janel.peterson@bcm.edu (J.A.P.); adahlgren@ucdavis.edu (A.R.D.) \\ enburns@ucdavis.edu (E.H.) \\ 2 Department of Veterinary Medicine and Epidemiology, University of California, Davis, CA 95616, USA; \\ mraleman@ucdavis.edu \\ * Correspondence: cjfinno@ucdavis.edu
}

Received: 21 September 2019; Accepted: 14 October 2019; Published: 16 October 2019

\begin{abstract}
Juvenile idiopathic epilepsy (JIE) is an inherited disease characterized by recurrent seizures during the first year of life in Egyptian Arabian horses. Definitive diagnosis requires an electroencephalogram (EEG) performed by a veterinary specialist. A recent study has suggested that a 19 base-pair deletion, along with a triple-C insertion, in intron five of twelve ( $\triangle 19$ InsCCC; chr20:29542397-29542425: GTTCAGGGGACCACATGGCTCTCTATAGA>TATCTTAAGACCC) of the Tripartite Motif-Containing 39-Ribonuclease p/mrp 21kDa Subunit (TRIM39-RPP21) gene is associated with JIE. To confirm this association, a new sample set consisting of nine EEG-phenotyped affected and nine unaffected Egyptian Arabian horses were genotyped using Sanger sequencing. There was no significant genotypic $(P=1.00)$ or allelic $(P=0.31)$ association with the $\triangle 19$ InsCCC variant and JIE status. The previously reported markers in TRIM39-RPPB1 are therefore not associated with JIE in well-phenotyped samples. The $\triangle 19$ InsCCC variant is a common variant that happens to be positioned in a highly polymorphic region in the Arabian breed.
\end{abstract}

Keywords: convulsion; electroencephalogram; equine; seizure

\section{Introduction}

Juvenile idiopathic epilepsy (JIE) is an inherited disease characterized by recurrent seizures during the first year of life in Egyptian Arabian horses. Recurrent seizures can lead to complications, including head injury and aspiration pneumonia. After the first year of life, seizure activity ceases [1]. There are no known environmental factors linked to the clinical presentations of JIE [2]. A definitive diagnosis of JIE requires an electroencephalogram (EEG) to confirm the presence of paroxysmal activity (sharp waves and spikes) supportive of seizures during clinical manifestations of epilepsy [3-5]. Due to the similarity with other genetically associated epileptic disorders in humans [6] and its occurrence in specifically the Egyptian Arabian breed, veterinary researchers postulate that the disease may have a genetic basis $[1,7,8]$. Interpretation of pedigree data suggests that JIE may be heritable with an autosomal dominant mode of inheritance (MOI) (unpublished data). Recently, a study was published documenting an intronic 19 base-pair deletion coupled with a triple-C insertion ( $\triangle 19$ InsCCC; chr20:29542397-29542425: GTTCAGGGGACCACATGGCTCTCTATAGA>TATCTTAAGACCC) in the TRIM39-RPP21 gene as associated with JIE [9]. The aim of this study was to confirm this association in a group of EEG-confirmed affected and veterinary-reported unaffected Egyptian Arabian foals. 


\section{Materials and Methods}

Eighteen Egyptian Arabian horses were used in this study (nine affected and nine unaffected horses). Affected horses were phenotyped via an EEG examination by a board-certified veterinary neurologist (M.A.) as previously described [1]. Unaffected horses were determined to have no history of seizures throughout the first year of life by both owner and veterinary reports. Genomic DNA of all horses was isolated from EDTA samples using a Wizard ${ }^{\circledR}$ Blood DNA extraction Kit (Promega, Madison, WI, USA). DNA samples were quantified with a QIAxpert (QIAGEN, Hilden, Germany) and diluted to $50 \mathrm{ng} / \mu \mathrm{l}$. Genotyping at the $\Delta$ 19InsCCC position was carried out using the previously reported primer set [9]. DNA was amplified using polymerase chain reaction (PCR) and product sizes were confirmed on a $1 \%$ agarose gel. As some reactions consistently showed secondary amplification products, two new primer sets were constructed with the use of Primer3Plus [10] and UCSC In-Silico PCR [11] programs (Table S1). Individual reactions were run at the following settings: $95^{\circ} \mathrm{C} \mathrm{for} 15 \mathrm{~m}$, followed by 35 cycles of $95^{\circ} \mathrm{C}$ for $30 \mathrm{~s}, 60{ }^{\circ} \mathrm{C}$ for $30 \mathrm{~s}, 72{ }^{\circ} \mathrm{C}$ for $30 \mathrm{~s}$, and $72{ }^{\circ} \mathrm{C}$ for $5 \mathrm{~m}$. Due to the polymorphic nature of this region, reactions were set up in a range of annealing temperatures $\left(58-67^{\circ} \mathrm{C}\right)$. An ExoSAP-IT PCR Product Cleanup Kit (Thermo Fisher Scientific, Waltham, MA, USA) was used on all amplified samples, and amplicons were sequenced at the UCDNA sequencing facility (Davis, CA, USA). Raw .abi sequencing files were viewed using SEQUENCHER ${ }^{\circledR}$ (Gene Codes, Ann Arbor, MI, USA), and genotypes were manually determined. Association testing with the JIE phenotype was performed using genotypic and allelic-based Fisher's exact tests, with $P<0.05$. The presence of $\triangle 19$ InsCCC indels were further screened using data from Arabian horses in NCBI's Sequence Read Archive (SRA) database (https://www.ncbi.nlm.nih.gov/sra).

\section{Results}

With the use of three primer sets, all samples were successfully genotyped. The region containing the $\triangle 19$ InsCCC variant is highly polymorphic; the presence of additional single nucleotide polymorphisms (SNPs) and/or indels at both 5' and 3' of the $\Delta 19$ InsCCC boundary made construction of specific primers difficult (Figure S1). Both JIE-affected and unaffected horses had varying numbers of additional variants in this region (Table S2).

Of the nine unaffected horses, five were homozygous wild-type, two were homozygous for the $\triangle 19$ InsCCC variant, and two were heterozygous. Of the nine EEG-confirmed affected horses, four were wild-type, and the remaining five were homozygous for the $\triangle 19$ InsCCC variant (Table 1). A genotypic Fisher's exact test, assuming an autosomal dominant MOI for the disease, revealed no association with the disease $(P=1.00)$. An allelic Fisher's exact test also showed no statistically significant association between the $\triangle 19$ InsCCC variant and the disease $(P=0.31)$. Within NCBI's SRA database of 384 mapped equine genomes, only three (ERX2235970, ERX2235963, and ERX1598843) were Arabian horses (lineages unknown), and all were homozygous wild-type at the $\triangle 19$ InsCCC locus.

Previous work postulated that the $\triangle 19$ InsCCC variant was positioned within an unannotated exon, rather than in an intronic region, and therefore led to changes in the coding sequence [9]. To test this hypothesis, EquCab2.0 alignment files from the Functional Annotation of the Animal Genome (FAANG) project were viewed at the $\triangle 19$ InsCCC locus using the Integrative Genomics Viewer (IGV) [12]. FAANG RNA-seq (https://www.ebi.ac.uk/ena/data/view/ERA148755) data revealed no expression at the $\triangle 19$ InsCCC position in any sequenced neural tissues (cerebellar vermis, frontal cortex, hypothalamus, lateral cerebellum, occipital cortex, parietal cortex, and retina).

Table 1. Egyptian Arabian population $(n=18) \Delta 19$ InsCCC genotypes.

\begin{tabular}{ccccc}
\hline Status & Homozygous $\boldsymbol{\Delta \text { 19InsCCC }}$ & Heterozygous & Homozygous Wild-Type & Total \\
\hline Unaffected JIE & 2 & 2 & 5 & 9 \\
Affected JIE & 5 & 0 & 4 & 9 \\
Total & 7 & 2 & 9 & 18 \\
\hline \multicolumn{4}{c}{$P<0.05$, Genotypic Fisher's $P=1.00$, Allelic Fisher's $P=0.31$} \\
\hline
\end{tabular}




\section{Discussion}

The data gathered in this study refutes the genetic association with the previously described $\triangle 19$ InsCCC variant and JIE. As the original study [9] defining this association was unable to confidently phenotype unaffected Arabian horses, this initial association may have been a false positive due to the prevalence of this variant in the Egyptian Arabian breed. Horses in the previous study were diagnosed with JIE based on veterinary observation alone, as EEG testing was not performed. Additionally, only one horse in the previous study could be confidently classified as a control [9]. In our study, JIE affected horses were phenotyped via EEG, and previous seizure events and unexplained trauma during the first year of life were well documented on all affected and unaffected horses. Importantly, four out of nine of our EEG-confirmed JIE-affected foals were wild-type for the putative variant, making it highly unlikely that the $\triangle 19$ InsCCC variant within TRIM39-RPP1 is causative for JIE. This is likely a common variant in the Arabian breed; however, genotyping of a random sample of Arabian horses is necessary to confirm this theory. Additionally, with FAANG horse transcriptome data, we were able to determine that the $\triangle 19$ InsCCC is within an intron of TRIM39-RPP1, as data revealed no expression at the $\triangle 19$ InsCCC position in any sequenced neural tissues from adult horses. However, the possibility remains that expression of this region may be specific to a certain developmental time point.

The region surrounding the $\triangle 19$ InsCCC variant within TRIM39-RPP1 was particularly challenging to genotype due to a high degree of polymorphism 3' of the indel (Table S2) as well as some additional variants $5^{\prime}$ of the indel. In particular, genotyping of heterozygotes led to mixed peaks shortly after the start of the indel. With such a high degree of polymorphism within this region, it is unlikely that this region would reliably contribute to the JIE phenotype.

In conclusion, due to the high frequency of the $\triangle 19$ InsCCC variant in both the JIE-affected and unaffected horses $(q=0.44)$, we exclude these indels to be causative of JIE. Further genetic evaluation of JIE, with genome-wide association studies, is required using EEG-confirmed samples.

Supplementary Materials: The following is available online at http://www.mdpi.com/2073-4425/10/10/816/s1, Table S1: Primers for Sanger sequencing of the $\triangle 19$ InsCCC region in TRIM39-RPP2, Table S2: Egyptian Arabian population ( $\mathrm{n}=18) \triangle 19$ InsCCC genotypes and additional variants, Figure S1: Variants of 3' within the TRIM39-RPP1 sequence, Figure S1: An example chromatogram from an affected (JIE4) and unaffected (JIE18) Egyptian Arabian foal within the $\triangle 19$ InsCCC region in TRIM39-RPP2. There is extensive variation $3^{\prime}$ of the $\triangle 19$ InsCCC region, as depicted by stars (SNPs) and triangles (indels).

Author Contributions: Conceptualization, V.N.R., J.A.P., A.R.D., E.N.H., and C.J.F.; methodology, V.N.R., J.A.P., A.R.D., E.N.H. and C.J.F.; validation, V.N.R., J.A.P., A.R.D., E.N.H., and C.J.F.; formal analysis, V.N.R., A.R.D and C.J.F.; investigation, V.N.R.; resources, M.A.; data curation, V.N.R.; writing—original draft preparation, V.N.R. and C.J.F.; writing-review and editing, V.N.R., M.A., J.A.P., A.R.D., E.N.H., and C.J.F.; project administration, J.A.P. and C.J.F.; funding acquisition, C.J.F. and M.A.

Funding: Support for this study was provided by the Center for Equine Health with funds provided by the State of California pari-mutuel fund and contributions by private donors. Support for CJF was provided by the NIH National Center Advancing Translational Sciences (NCATS), Grant/Award Number: L40 TR001136 (CJF).

Conflicts of Interest: The authors declare no conflict of interest.

\section{References}

1. Aleman, M.; Gray, L.C.; Williams, D.C.; Holliday, T.A.; Madigan, J.E.; Lecouteur, R.A.; Magdesian, K.G. Juvenile Idiopathic Epilepsy in Egyptian Arabian Foals: 22 Cases (1985-2005). J. Vet. Intern. Med. 2006, 20, 1443-1449.

2. Sengoku, A. The Contribution of J.H. Jackson to Present-Day Epileptology. Epilepsia 2002, 43, 6-8. [CrossRef] [PubMed]

3. Berg, A.T.; Berkovic, S.F.; Brodie, M.J.; Buchhalter, J.; Cross, J.H.; Boas, W.V.E.; Engel, J.; French, J.; Glauser, T.A.; Mathern, G.W.; et al. Revised terminology and concepts for organization of seizures and epilepsies: Report of the ILAE Commission on Classification and Terminology, 2005-2009. Epilepsia 2010, 51, 676-685. [CrossRef] [PubMed]

4. Shorvon, S.D. The etiologic classification of epilepsy. Epilepsia 2011, 52, 1052-1057. [CrossRef] [PubMed] 
5. Trinka, E.; Cock, H.; Hesdorffer, D.; Rossetti, A.O.; Scheffer, I.E.; Shinnar, S.; Shorvon, S.; Lowenstein, D.H. A definition and classification of status epilepticus - Report of the ILAE Task Force on Classification of Status Epilepticus. Epilepsia 2015, 56, 1515-1523. [CrossRef] [PubMed]

6. Hirose, S.; Mitsudome, A.; Okada, M.; Kaneko, S. Genetics of Idiopathic Epilepsies. Epilepsia 2005, 46, $38-43$. [CrossRef] [PubMed]

7. Lacombe, V.A. Seizures in horses: Diagnosis and classification. Vet. Med. 2015, 6, 301-308. [CrossRef] [PubMed]

8. Gardiner, M. Genetics of Idiopathic Generalized Epilepsies. Epilepsia 2005, 46, 15-20. [CrossRef] [PubMed]

9. Polani, S.; Dean, M.; Lichter-Peled, A.; Hendrickson, S.; Tsang, S. Sequence Variant in the TRIM39-RPP21 Gene Readthrough is Shared across a Cohort of Arabian Foals Diagnosed with Juvenile Idiopathic Epilepsy. J. Gene Mutat 2018, 1, 103.

10. Untergasser, A.; Cutcutache, I.; Koressaar, T.; Ye, J.; Faircloth, B.C.; Remm, M.; Rozen, S.G. Primer3-new capabilities and interfaces. Nucleic Acids Res. 2012, 40, e115. [CrossRef] [PubMed]

11. Kent, W.J.; Sugnet, C.W.; Furey, T.S.; Roskin, K.M.; Pringle, T.H.; Zahler, A.M.; Haussler, D. The human genome browser at UCSC. Genome Res. 2002, 12, 996-1006. [CrossRef] [PubMed]

12. Robinson, J.T.; Thorvaldsdóttir, H.; Winckler, W.; Guttman, M.; Lander, E.S.; Getz, G.; Mesirov, J.P. Integrative Genomics Viewer. Nat. Biotechnol. 2011, 29, 24-26. [CrossRef] [PubMed]

(C) 2019 by the authors. Licensee MDPI, Basel, Switzerland. This article is an open access article distributed under the terms and conditions of the Creative Commons Attribution (CC BY) license (http://creativecommons.org/licenses/by/4.0/). 